\title{
Analysis of the Temporal Behaviors of the Carrier-Mediated Membrane Transport by Optimal Control Theory
}

\author{
Member Hirohumi Hirayama (Asahikawa Medical College) \\ Non-member Toshihiro Nishimura (Oita University) \\ Member Yuzo Fukuyama (Asahikawa Medical College)
}

\begin{abstract}
Theoretical analysis was performed on the carrier-mediated solute transport across the biological membrane based on optimal control principle that minimizes the rate of changes in concentrations of the species in combination with energy to drive the system. Experimental data for temporal change in carrier mediated transport could be simulated by the present model with regulating weighting coefficients. Changes in concentrations of the species due to the increase in rate constants could be explained by the compensatory action of the optimal control mechanism. The present study must be available for evaluation of the optimality in carrier mediated membrane transport.
\end{abstract}

key words: Membrane, Carrier, Transport, Solute, Optimal Control, Performance Function

\section{Introduction}

In biological systems, many materials are transported into intra cellular space through the biological membrane. There are three mechanisms of the transport across the membrane ${ }^{11}$,

(1) Passive transport: simple diffusion, diffusion through selective channel and facilitated diffusion $^{(2)(3)}$.

(2) Active transport: the primary active transport such as Ion transport, carrier protein dependent transport, secondary active transport such as co-transport or symport of Ions, anti port and exchange transport ${ }^{(4)(5)}$ and

(3) Group tans location

Regardless of these mechanisms, ultimately they are performed by a solute specific carrier protein. Once, the solute has connected with the carrier protein and formed a complex, conformational changes occurr in the carrier protein. As a result, a solute is transported across the membrane as a solute-carrier complex.

Considerable amounts of investigations have been accumulated and minute mechanisms are disclosed. However, there has been any study for the control strategy of membrane transport particularly from the stand point of economical operation.
In the present investigation, we propose a mathematical model of carrier mediated membrane transport based the optimal control mechanism. The fundamental concept is that the solute is transported across the membrane upon minimizing the energy and achieving the least change state without retention or depletion of all the participated species at any step of the transport process.

We show successful simulation for biological experimental result, the optimized time courses of concentrations of carrier-solute complexes and the influences of elevated rate constants.

\section{Mathematical modeling}

\subsection{Modeling of the carrier-mediated transport} across the membrane

A generalized model of the carrier mediated transport across the biological membrane is shown in fig. 1. The model consists of 4 steps of transport ${ }^{(5)}$ as followings: (1) The solutes in the extra cellular space $\left(S_{1}\right)$ bind to the free carriers $\left(C_{1}\right)$ on the outer surface of the mem. brane facing to the extra-cellular space.

$$
\mathrm{C}_{1}+\mathrm{S}_{1} \underset{k_{2}}{\stackrel{k_{1}}{\rightleftarrows}} \mathrm{CS}_{1}
$$

(2) The carrier-solute complex $\left(\mathrm{CS}_{1}\right)$ moves from the outer surface of the membrane to the inmer surface of 
S1 (extra cellular space)

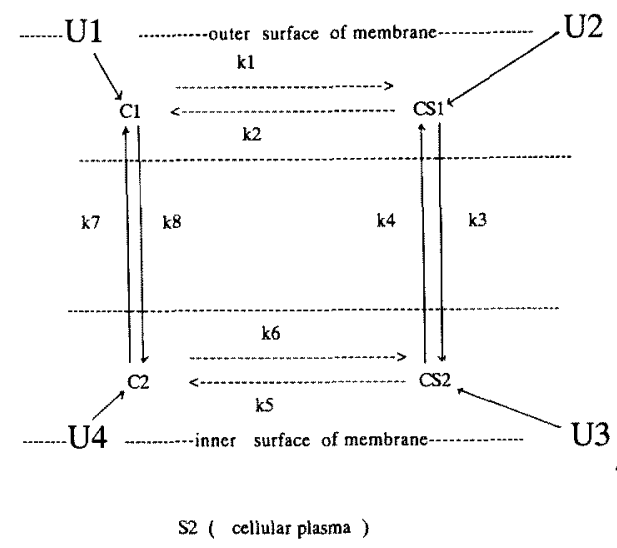

Thick arrows indicate transport from extra cellular space to cellular plasma and thin arrows are those for reverse direction. $U_{1}, U_{2}, U_{3}$ and $U_{4}$ are the control inputs for $C_{1}$, $\mathrm{CS}_{1}, \mathrm{CS}_{2}$ and $\mathrm{C}_{2}$ respectively.

Fig. 1. The schema of model of carrier-mediated membrane transport.

the membrane $\left(\mathrm{CS}_{2}\right)$ facing to the cellular plasma.

$$
\mathrm{CS}_{1} \underset{k_{4}}{\stackrel{k_{3}}{\rightleftarrows}} \mathrm{CS}_{2}
$$

(3) The carrier-solute complex $\left(\mathrm{CS}_{2}\right)$ on the inner surface of the membrane releases the solutes to the cellular plasma.

$$
\mathrm{CS}_{2} \underset{k_{6}}{\stackrel{k_{5}}{\rightleftarrows}} \mathrm{S}_{2}+\mathrm{C}_{2}
$$

(4) The solute free carriers $\left(C_{2}\right)$ on the inner surface of the membrane return to the outer surface of the membrane $\left(C_{1}\right)$. This process expresses the recycling phase of the carrier.

$$
\mathrm{C}_{2} \underset{k_{8}}{\stackrel{k_{7}}{\longrightarrow}} \mathrm{C}_{1}
$$

where

$\left[S_{1}\right]$ : the concentration of the solute per unit volume of the extra-cellular space.

$\left[S_{2}\right]$ : the concentration of the solute per unit volume of the cellular plasma.

$\left[C_{1}\right]$ : the concentration of free carrier per unit area of the outer surface of the membrane.

$\left[\mathrm{C}_{2}\right]$ : the concentration of free carrier per unit area of the inner surface of the membrane.

$\left[\mathrm{CS}_{1}\right]$ : the concentration of the solute-carrier complex per unit area of the outer surface of the membrane.

$\left[\mathrm{CS}_{2}\right]$ : the concentration of the solute-carrier complex per unit area of the inner surface of the mem. brane. $\mathrm{kn}$ is a rate constant of the reaction per unit time per unit area of the membrane. $k_{1}, k_{3}, k_{5}$ and $k_{7}$ are those when the solutes are carried into the cellular plasma. $k_{2}$, $k_{4}, k_{6}$ and $k_{8}$ are those of transport in the reverse direc. tion. The instantaneous changes of the concentrations of the species are

$$
\begin{aligned}
& d\left[\mathrm{C}_{1}\right] / d t=k_{2}\left[\mathrm{CS}_{1}\right]+k_{7}\left(\mathrm{C}_{2}\right]-k_{8}\left[\mathrm{C}_{1}\right]-k_{1}\left[\mathrm{C}_{1}\right]\left[\mathrm{S}_{1}\right]
\end{aligned}
$$

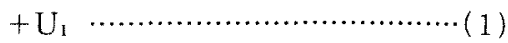

$$
\begin{aligned}
& d\left[\mathrm{CS}_{1}\right] / d t=k_{1}\left[\mathrm{C}_{1}\right]\left[\mathrm{S}_{1}\right]+k_{4}\left(\mathrm{CS}_{2}\right)-k_{3}\left[\mathrm{CS}_{1}\right] \\
& -k_{2}\left[\mathrm{CS}_{1}\right]+\mathrm{U}_{2} \\
& d\left[\mathrm{CS}_{2}\right] / d t=k_{3}\left[\mathrm{CS}_{1}\right]+k_{6}\left[\mathrm{C}_{2}\right]-\left(k_{4}+k_{5}\right)\left[\mathrm{CS}_{2}\right]+\mathrm{U}_{3} \\
& \text { …............................... (3) } \\
& d\left[\mathrm{C}_{2}\right] / d t=k_{5}\left[\mathrm{CS}_{2}\right]+k_{8}\left[\mathrm{C}_{1}\right]-\left(k_{7}+k_{6}\right)\left[\mathrm{CS}_{2}\right]+\mathrm{U}_{4} \\
& \text {.............................. (4) } \\
& d\left[\mathrm{~S}_{2}\right] / d t=k_{5}\left[\mathrm{CS}_{2}\right]
\end{aligned}
$$

where $\mathrm{U} n(n=1,2,3,4)$ are the control inputs. The solutes in the extra-cellular space exist in sufficient amount. So we set $\left[S_{1}\right]$ as a constant. By the law of conservation,

$$
\left[\mathrm{C}_{t}\right]=\left[\mathrm{C}_{1}\right]+\left[\mathrm{C}_{2}\right]+\left[\mathrm{CS}_{1}\right]+\left[\mathrm{CS}_{2}\right]
$$

where $\mathrm{C}_{t}$ is the total concentration of the carrier per unit area of the membrane. By converting the concen. trations of these species to state variables,

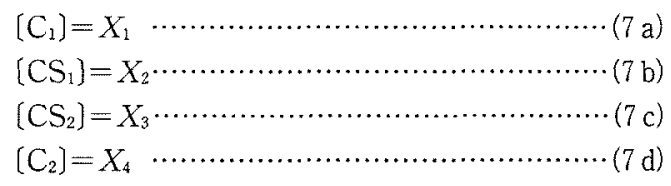

and

$$
\left[\mathrm{C}_{t}\right]=X_{0}
$$

Substituting $X_{3}=X_{0}-X_{1}-X_{2}-X_{4}$ into the equation (2), (3) and (4) the following are achieved:

$d X_{1} / d t=X_{1}\left(-k_{8}-k_{1}\left(\mathrm{~S}_{1}\right]\right)+k_{2} X_{2}+k_{7} X_{4}+\mathrm{U}_{1}$

$$
\begin{aligned}
d X_{2} / d t= & k_{1}\left[\mathrm{~S}_{1}\right] X_{1}+\left(-k_{2}-k_{3}\right) X_{2} \\
& +k_{4}\left(X_{0}-X_{1}-X_{2}-X_{4}\right)+\mathrm{U}_{2} \cdots \cdots \\
d X_{4} / d t= & k_{8} X_{1}+\left(k_{5}-k_{7}-k_{6}\right)\left(X_{0}-X_{1}-X_{2}\right. \\
& \left.-X_{4}\right)+\mathrm{U}_{4} \quad \cdots \cdots \cdots \cdots \cdots \cdots \cdots \cdots \cdots \cdots \cdots \cdots \cdots \cdots \cdots \cdots \cdots \\
d X_{3} / d t= & -d X_{1} / d t-d X_{2} / d t-d X_{4} / d t
\end{aligned}
$$

\subsection{Setting the control inputs and their biological significance}

$\mathrm{U}_{1}$ is the control input for production of carrier protein. Because production of the carrier protein, $\mathrm{C}_{1}$ has to be controlled economically to achieve the optimal tansport state. This process is ultimately the result of the translation and protein synthesis on the messenger RNA. Its over production reduces the economical trans port because of the unbalance of demand (solute that has to be carried) and supply (carrier production). On the contrary, its insufficient production makes the solute to accumulate abnormally in the extra cellular space 
which in turn disturbs the physiological cellular function. Thus, we set $U_{1}$ as the control input that has to be controlled optimally.

$\mathrm{U}_{3}$ is the control input for releasing the solutes at the inner surface of the membrane from the carrier-solute complex $\mathrm{CS}_{2}$. Essentially, this process relates to phosphorylation which is controlled by many humoral fac. tors such as adrenalin.

$\mathrm{U}_{2}$ is the control input for transporting the carrier. solute complex $\mathrm{CS}_{1}$ across the membrane to the inner surface of the membrane. For this transportation, certain amount of biological energy is required to overcome the electro-chemical potential gradient across the cellular membrane ${ }^{(2)(6)(7)}$. When the solutes have charges, the energy for the transport is expressed by

$$
\Delta \mu \mathrm{S}=R T \operatorname{In}([\mathrm{S}] \text { in } /[\mathrm{S}] \text { out })+n F \Delta \phi
$$

where

$\Delta \mu \mathrm{s}$ : the electro chemical potential gradient of the solutes across the membrane,

$R$ : the Gas coefficient, $T$ : the absolute temperature, $F$ : the Farady coefficient, $\Delta \phi$ : the difference of the electrical potential across the membrane,

$n$ : the valent of an electron and

[S] in (S) out: the concentrations of the solutes in cellular plasma and extra cellular space.

To transport charged solutes by the carriers, this amount of energy has to be supplied. The most typical example is the active transport system of ions by ATP (Adenosine Tri Phosphate) -ase ${ }^{(8)}$. The carriers transport ions against the chemical gradient by utilizing the energy described above by hydrolysis of ATP. Therefore, utilizing the energy derived from ATP per unit time per unit area of the membrane was set as the control input, $U_{2}$ for the transport across the membrane. A similar explanation is given for $\mathrm{U}_{4}$.

\subsection{Setting the cost function for evaluating performance of the transport}

We propose the equation(13) for the cost function to evaluate the performance of the carrier mediated trans. port.

$$
\begin{aligned}
J= & \int_{0}^{t e}\left[\sum_{n=1}^{4} \partial_{n}\left(\partial X_{n} / \partial t\right)^{2}+\sum_{n=1}^{4} \gamma_{n}\left(X_{n}-A_{n}\right)^{2}\right. \\
& \left.+\sum_{n=1}^{4} \beta_{n} \mathrm{U}_{n}^{2}\right] d t \ldots \ldots \ldots \ldots \ldots \ldots \ldots \ldots \ldots \ldots \ldots \ldots \ldots \ldots \ldots \ldots \ldots \ldots
\end{aligned}
$$

We assert that the optimal carrier-mediated transport is achieved when this cost function has been minimized during $[0, t e]$. The significance of the cost function ${ }^{(11)(12)}$ are

(1) The first 4 terms describe to minimize the rates of temporal changes in the concentrations of species,

\section{$\left[\mathrm{C}_{1}\right],\left[\mathrm{C}_{2}\right],\left[\mathrm{CS}_{1}\right],\left[\mathrm{CS}_{2}\right]$}

(2) The second 4 terms describe to minimize the deviations from the target concentrations, $A_{n}(n=1,2,3$ and 4) for the species, $\left[\mathrm{C}_{1}\right],\left[\mathrm{C}_{2}\right],\left[\mathrm{CS}_{1}\right],\left[\mathrm{CS}_{2}\right]$ and

(3) The last 4 terms express to minimize the consumption of the control inputs to drive the system.

The most important aspect of the transport is to carry the solutes smoothly without abnormal congestion (over functioning) nor depletion (in the vacant state) of solute, carrier and / or carrier-solute complex at any step in the transport process.

Minimizing the rates of temporal changes in concentrations prevents excessive rapid accumulation of solutes and / or carrier-solute complex and results in a smooth transport. If any one of these 4 species increased excessively or depleted completely, economical transport would be disturbed.

For example, if $\mathrm{CS}_{1}$ increased rapidly to an excess amount, $\mathrm{CS}_{1}$ would accumulate wastefully on the outer surface of the membrane unless control input works optimally so as to transport the surplus $\mathrm{CS}_{1}$ rapidly to the inner surface. As a result, the solutes and the carriers would congest abnormally. These surplus $\mathrm{CS}_{1}$ would be obstacles for free carriers to link with solute to form the complex $\mathrm{CS}_{1}$. Moreover, excess $\mathrm{CS}_{1}$ will drive the reaction of $\mathrm{C}_{1}+\mathrm{S}_{1} \rightarrow \mathrm{CS} 1$ to reverted direction and surplus $C_{1} S_{1}$ would be dissociated to $C_{1}$ and $S_{1}$. Thus, minimizing the rates of the temporal changes in the concentrations of the components is an important condition for achieving economical transport.

The second four terms indicate the space in the membrane which solutes, carriers and carrier-solute complexes can occupy per unit area of the membrane has their limitations.

Minimizing the control inputs $\mathrm{U}_{n}(n=1,2,3,4)$ is vital, because the carriers have to be produced economically (demand and supply matching) and the transport across the membrane against the electro chemical potential gradient has to utilize biological energy from ATP (adenosine tri phosphate) effectively. Thus, saving the energy derived from ATP results in an economical operation of the transport.

The weighting coefficients $\alpha_{n}, \beta_{n}$ and $\gamma_{n}(n=1,2,3$ and 4) measure the relative magnitude of minimizing the changes of components timed by those weighting coefficients.

\subsection{The optimal solution}

Substituting the equations (8) to (11) into the cost function and rearranging, we have Hamiltonian 


$$
\begin{aligned}
H= & d_{1} X_{1}^{2}+d_{2} X_{2}^{2}+d_{3} X_{4}^{2}+d_{4} \mathrm{U}_{1}^{2}+d_{5} \mathrm{U}_{2}^{2} \\
& +d_{6} \mathrm{U}_{3}^{2}+d_{7} \mathrm{U}_{4}^{2}+d_{8} X_{1} X_{2}+d_{9} X_{1} X_{4} \\
& +d_{10} X_{2} X_{4}+d_{11} X_{1} \mathrm{U}_{1}+d_{12} X_{2} \mathrm{U}_{2} \\
& +d_{13} X_{4} \mathrm{U}_{1}+d_{14} X_{1} \mathrm{U}_{2}+d_{15} X_{2} \mathrm{U}_{2} \\
& +d_{16} X_{4} \mathrm{U}_{2}+d_{17} X_{1} \mathrm{U}_{3}+d_{18} X_{2} \mathrm{U}_{3} \\
& +d_{19} X_{4} \mathrm{U}_{3}+d_{20} X_{1} \mathrm{U}_{4}+d_{21} X_{2} \mathrm{U}_{4} \\
& +d_{22} X_{4} \mathrm{U}_{4}+d_{23} X_{1}+d_{24} X_{2}+d_{25} X_{4} \\
& +d_{26} \mathrm{U}_{2}+d_{27} \mathrm{U}_{3}+d_{28} \mathrm{U}_{4} \\
& +p_{1}\left(a_{1} X_{1}+a_{2} X_{2}+a_{3} X_{4}+\mathrm{U}_{1}\right) \\
& +p_{2}\left(a_{4} X_{1}+a_{5} X_{2}+a_{6} X_{4}+a_{7}+\mathrm{U}_{2}\right) \\
& +p_{4}\left(a_{12} X_{1}+a_{13} X_{2}+a_{14} X_{4}+a_{15}+\mathrm{U}_{4}\right)
\end{aligned}
$$

where $d_{n}$, an are constants composed of the system parameters and the weighting coefficients. $p_{1}, p_{2}$ and $p_{4}$ are the adjoin variables for $X_{1}, X_{2}$ and $X_{4}$. The optimal control is obtained by differentiating Hamiltonian function with respect to $\mathrm{U}_{n}$.

$$
\partial H / \partial \mathrm{U}_{n}=0
$$

Thus,

$$
\begin{aligned}
& \partial H / \partial \mathrm{U}_{1}=2 d_{4} \mathrm{U}_{1}+d_{11} X_{1}+d_{12} X_{2}+d_{13} X_{4}+p_{1} \\
& \text {......................... (16 a) } \\
& \partial H / \partial \mathrm{U}_{2}=2 d_{5} \mathrm{U}_{2}+d_{14} X_{1}+d_{15} X_{2}+d_{16} X_{4}
\end{aligned}
$$

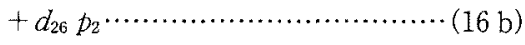

$$
\begin{aligned}
& \partial H / \partial \mathrm{U}_{3}=2 d_{6} \mathrm{U}_{3}+d_{17} X_{1}+d_{18} X_{2}+d_{19} X_{4}+d_{27} \\
& \text { …............................ }(16 \mathrm{c}) \\
& \partial H / \partial \mathrm{U}_{4}=2 d_{7} \mathrm{U}_{4}+d_{20} X_{1}+d_{21} X_{2}+d_{22} X_{4}
\end{aligned}
$$

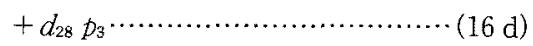

The optimal $\mathrm{U}_{n}$ are

$$
\begin{aligned}
& \mathrm{U}_{1}=d_{29} X_{1}+d_{30} X_{2}+d_{31} X_{4}+d_{32} p_{1} \cdots \cdots \cdots \cdots(17 \mathrm{a}) \\
& \mathrm{U}_{2}=d_{33} X_{1}+d_{34} X_{2}+d_{35} X_{4}+d_{35} p_{2}+d_{37} \cdots \cdots(17 \mathrm{~b}) \\
& U_{3}=d_{38} X_{1}+d_{39} X_{2}+d_{40} X_{4}+d_{41} \cdots \cdots \cdots \cdots \cdots(17 \mathrm{c}) \\
& \mathrm{U}_{4}=d_{42} X_{1}+d_{43} X_{2}+d_{44} X_{4}+d_{45} p_{3}+d_{46}
\end{aligned}
$$

The optimal differential equations for the state variables are modified to

$$
\begin{aligned}
& X_{1}^{\prime}=a_{1} X_{1}+a_{2} X_{2}+a_{3} X_{4}+d_{29} X_{1}+d_{30} X_{2} \\
& +d_{31} X_{4}+d_{32} p_{1} \quad \cdots \cdots \cdots \cdots \cdots \cdots \cdots \cdots \cdots(18 \mathrm{a}) \\
& X_{2}^{\prime}=a_{4} X_{1}+a_{5} X_{2}+a_{6} X_{4}+d_{33} X_{1}+d_{34} X_{2} \\
& +d_{35} X_{4}+d_{36} p_{2}+d_{37} \cdots \cdots \cdots \cdots \cdots \cdots \cdots(18 \mathrm{~b}) \\
& X_{3}^{\prime}=a_{12} X_{1}+a_{13} X_{2}+a_{14} X_{4}+d_{42} X_{1}+d_{43} X_{2} \\
& +d_{44} X_{4}+d_{45} p_{3}+d_{46}
\end{aligned}
$$

The differential equations for the co-state variables are obtained by differentiating Hamiltonian with respect to individual state variable.

$$
\begin{aligned}
& p_{1}^{\prime}=-\partial H / \partial X_{1} \\
& =h_{1} X_{1}+h_{2} X_{2}+h_{3} X_{4}+h_{4} p_{6}+h_{5} p_{2}+h_{6} p_{4}+h_{7} \\
& \text {........................... (19 a) } \\
& p_{2}^{\prime}=-\partial H / \partial X_{2} \\
& =h_{8} X_{1}+h_{9} X_{2}+h_{10} X_{4}+h_{11} p_{1}+h_{12} p_{2}+h_{13} p_{4}+h_{14}
\end{aligned}
$$

$$
\begin{aligned}
p_{3}^{\prime} & =-\partial H / \partial X_{3} \\
& =h_{14} X_{1}+h_{15} X_{2}+h_{16} X_{4}+h_{17} p_{1}+h_{18} p_{2}+h_{19} p_{4}
\end{aligned}
$$

Consequently, we have six simultaneous linear differential equations.

\subsection{Setting system parameters}

A carrier protein has at least two subunits for binding to solutes ${ }^{(1)(2)}$. Lied and Stein ${ }^{(14)}$ proposed a model of a carrier protein which consisted of two subunits. Thus, probabilistically, the rate constant can be 0.5 . Experimental analysis ${ }^{(15)}$ of glucose accumulation by an $H$ ion-coupled active transport revealed that in a stable state, the fraction of co-transporter intermediates (corresponding to solute-carrier complexes) ranged from 0 . 01 to 0.78 . In the facilitated diffusion of vehicles ${ }^{(16)}$, rate constant ranged 0.5 to 0.8 . Therefore, the rate constant differs among the different kind of transport and materials. The present study, however, analyzes a general case and not a special one. For the convenience, we assumed that the transport starts from the steady and completely balanced state that had been achieved aprior. This means that species have distributed evenly at all the sides of membrane when the transport stars to operate. This feature is characterized by the equivocal initial condition (equation $17 \mathrm{a}$ ). Thus, we set $k_{1}=k_{3}=k_{5}$ $=k_{7}=0.5^{(17)}$.

We analyze the situation when the import exceeds the export, so we set $k_{2}=k_{4}=k_{6}=k_{8}=0.01$. The target concentrations of the species were set to $A_{n}(n=1,2,3$ and 4$)=0.5$. Because we have assumed the complete equilibrium state at the onset of new transport cycle, the final concentrations of all the species $\left(X_{n}(t e)\right)$ have to be equal to the target concentrations $\left(A_{n}\right)$. The total amount of the solutes and carriers are set to $S_{1}=5.0$ and $\mathrm{C}_{t}=5.0$ for the simplicity.

The weighting coefficients were set to be unity $\alpha_{n}=\beta_{n}$ $=\gamma_{n}=1.0(n=1,2,3,4)$ at the standard state and being regulated to simulate the experimental data. Consequently, the initial and final concentrations of the components were set $^{(17)}$ as

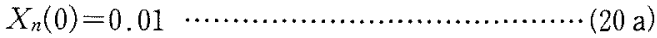

$$
\begin{aligned}
& X_{n}(t e)=0.5 \quad(n=1,2,4)
\end{aligned}
$$

The non zero conditions are indispensable for evoking the transport. For, example, Sodium-Potassium pump operates only when certain initial amounts of $\mathrm{Na}$ and $\mathrm{K}$ exist $^{(3)(4)}$. The terminal states $X_{n}(t e)$ were set to equiva. lent to the target concentration An because the incon. sistency between the terminal and target concentrations indicates that the system can not achieve the object of the transport. 
Mathematically, the present problem can be reduced to a two points boundary values problem. This formalization is important to describe the recycling nature of the carrier transport. This process is achieved particularly by setting the terminal condition which links to an initial for subsequent new transport cycle. For the simplicity, we set $t e=1,0$.

Optimal time courses of $X_{1}, X_{2}$ and $X_{4}$ were obtained by solving equations (18) and (19) under the boundary conditions (20) by numerical method.

\section{Results}

\subsection{Simulation of the reported experimental data}

Fig. 2. shows simulating the reported experimental data for transient changes of the concentrations of $\mathrm{C}_{1}$, $\mathrm{CS}_{1}$ and $\mathrm{C}_{2}$ of $\mathrm{Na} /$ glucose co-transport ${ }^{(18)}$. The parameters used for the simulation were shown in Table 1.

Table 1. Parameters for simulation.

1. Rate Constants

$k_{1}=120, k_{2}=23, k_{3}=k_{4}=0.9$

$k_{5}=40, k_{8}=3.9, k_{7}=8.9, k_{8}=1.45$

2. Weighting Coefficients

$\alpha_{1}=1.0 \quad \alpha_{2}=500 \quad \alpha_{3}=1.0 \quad \alpha_{4}=200$

$\beta_{1}=10 \quad \beta_{2}=1 \quad \beta_{3}=20 \quad \beta_{4}=30$

$\gamma_{1}=1 \quad \gamma_{2}=1 \quad \gamma_{3}=4 \quad \gamma_{5}=1$

3. Initial Conditions

$C_{1}(0)=0.1 \quad C_{1}(0)=0.2 \quad C_{2}(0)=0.2$

4. Terminal Conditions

$C_{1}(t e)=0.01 \quad C_{1}(t e)=0.1 \quad C_{2}(t e)=0.63$
There were ineviable discrepancies with experimental data.

3.2 Influences of increased rate constants on the optimized temporal changes in concentrations of the species

The rate constants, $k_{3}, k_{5}$ and $k_{7}$ were increased ten folds while $k_{1}$ was two fold. With increases in $k_{5}$ and $k_{7}$, $C_{1}$ increased rapidly [Fig. 3(a)]. Increases in these coefficients accelerate the transport of $\mathrm{CS}_{2}$ and $\mathrm{C}_{2}$. As a result, $\mathrm{CS}_{2}$ and $\mathrm{C}_{2}$ would be depleted rapidly. Thus, to compensate such reductions of $\mathrm{CS}_{2}$ and $\mathrm{C}_{2}, \mathrm{C}_{1}$ must be supplied as soon as possible. As a result, $C_{1}\left(: X_{1}\right)$ was controlled to increase. This reaction can be attributed to the operation of the optimal control that prevent $\mathrm{Cl}$ from abnormal decreasing. Similar pattern could be observed for $\mathrm{CS}_{1}\left(: X_{2}\right)$ but lesser effect by $k_{7}$ which locates away from $\mathrm{CS}_{1}$. The increase in $k_{1}$ reduced $\mathrm{C}_{1}$. This was due to an enhanced linkage of $C_{1}$ to $C S_{1}$. The reduction of $\mathrm{CS}_{1}\left(: X_{2}\right)$ [Fig. $3(\mathrm{~b})$ ] as a response to increase in $k_{1}$ and $\mathrm{C}_{2}\left(: X_{4}\right)$ [Fig. $3(\mathrm{c})$ ) as response to increase in $k_{5}$ were interesting. One possible explanation is that the optimal control operates to avoid the rapid changes in concentrations of $\mathrm{CS}_{1}$ and $\mathrm{C}_{2}$ induced by an accelerated transportations toward $\mathrm{CS}_{1}$ and $\mathrm{C}_{2}$. As a result, they have been reduced rather than being in creased. For CS [Fig. 3(b) ), increase in $k 5$ induced rapid increase. This must also be the compensatory operation of the optimal control mechanism to minimize

$[\mathrm{C} 1],[\mathrm{CS} 1],[\mathrm{C} 2]$

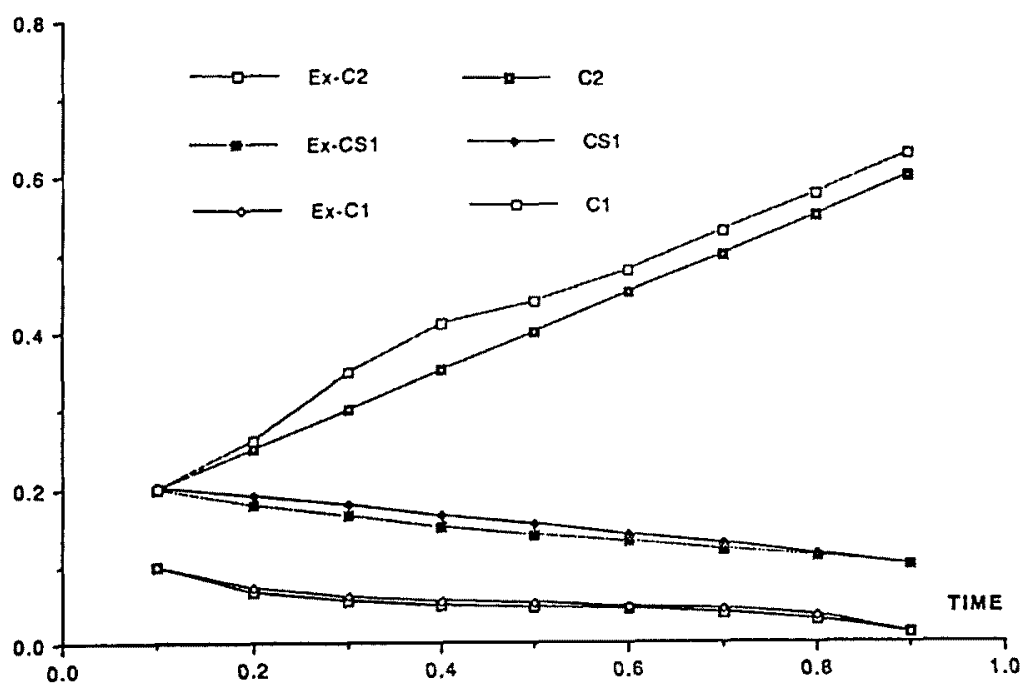

Simulation was performed by regulating the weighting coefficients and using reported experi. mental data of rate constants. The time courses of concentrations of $\mathrm{C}_{1}, \mathrm{CS}_{3}$ and $\mathrm{C}_{2}$ of reported experimental data were denoted by $\mathrm{Ex}-\mathrm{C}_{1}, \mathrm{Ex} \cdot \mathrm{CS}_{1}$ and $\mathrm{Ex}-\mathrm{C}_{2}$ and those without Ex are the theoretical results obtained by the optimized model.

Fig. 2. Simulation of experimental data 


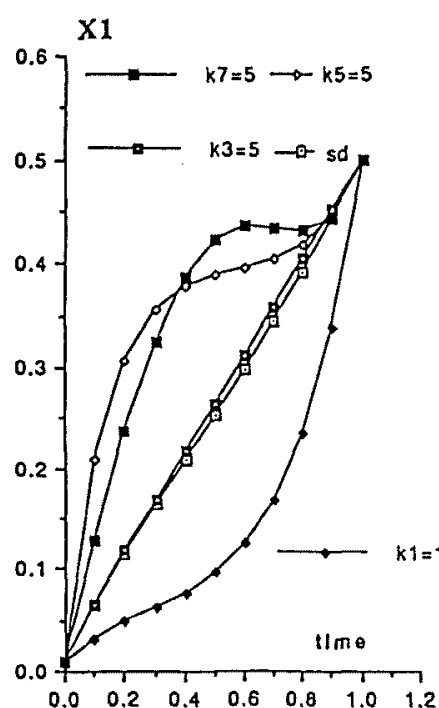

(a)

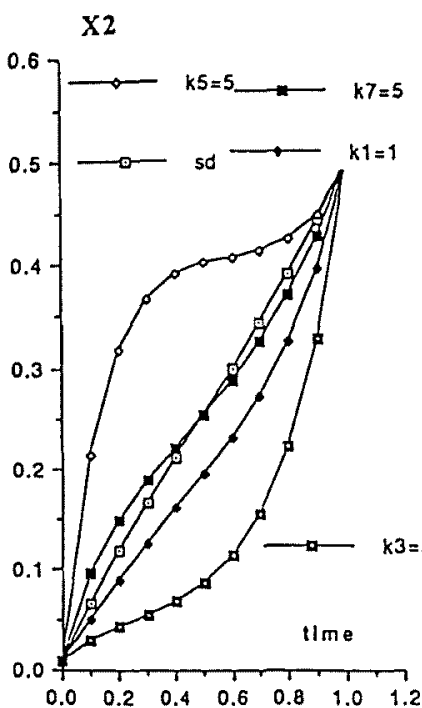

(b)

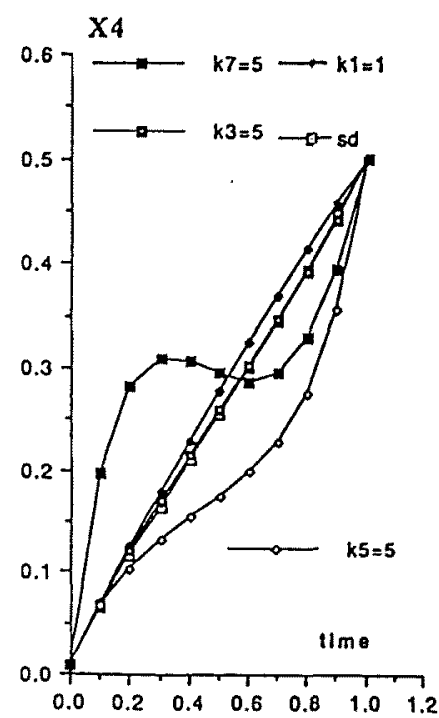

(c)

Fig. 3. The optimized time courses of concentrations of $X_{1}\left(=\left[\mathrm{C}_{1}\right]\right), X_{2}\left(=\left[\mathrm{CS}_{1}\right]\right)$, and $X_{4}\left(=\left[\mathrm{C}_{2}\right]\right)$ at the standard level of rate constants $k_{n}$ (sd in figures, $k_{3}=k_{5}=k_{7}=0.5$ and $k_{2}=k_{4}=k_{6}=k_{8}=0.01$ ). Rate constants were increased isolatedly and the time course of elevated rate constants were indicated by $k_{3}$, $k_{5}, k_{7}=5.0, k_{6}=1.0$. All other rate constants were set to the standard level. The transporting time is normalized to 1.0 .

the reduction of $\mathrm{CS}_{2}$ due to enhanced conversion to $\mathrm{C}_{2}$. There was, however, little effect by $k_{7}$ on $\mathrm{CS}_{1}$. Similar patterns could be observed in $\mathrm{C}_{2}$ [Fig. $\left.3(\mathrm{c})\right]$ with less obvious effects by $k_{n}$.

\section{Discussion}

The present paper proposed a generalized model of carrier mediated transport across the biological membrane. Particular emphasizes have been put on

(1) It is based on the optimal control principle. There has been no strategic inventigation although we intuitively understood that the biological system prefer to work in the least change state, namely Homeostasis.

(2) Recycling nature to recruit free carrier on the inner surface of the membrane to free carrier on the outer surface. This property can be expressed by the two points boundary value problem.

We have shown that the present theory can describe the physiological carrier mediated transport by presenting successful simulation of experimental data.

The rate constant is one of the most critical factor that determines the temporal behaviors of the transport across the membrane. From experimental results ${ }^{(19)(20)}$, association-dissociation reactions on membrane surfaces were faster than the transport across the membrane. However, data on the transport of L-Leucine into human erythrocytes at $25^{\circ} \mathrm{C}$ showed ${ }^{(17)}$ that at a stable state, the carrier is equally distributed at any site of the membrane. Since we analyze the steady state in which the transport is stable, we set $k_{1}, k_{3}, k_{5}$ and $k_{7}$ equal to 0 . 5.

There are many other biological features of the rate constant, for example, the rate of the binding of solutes to the carriers enhances the rate of the transport across the membrane ${ }^{(17)}$, saturation phenomenon ${ }^{(2)}$, competition of binding sites with antagonist ${ }^{(2)}$ and voltage dependent co-transport with ions ${ }^{(17)}$. Such functional settings make the present problem insolvable ${ }^{(13)}$, we set the present rate constants as independent ones.

\section{Conclusion}

(1) A theoretical model of carrier-mediated transport across the biological membrane based on the optimal control principle can reconstruct physiological experimental data for membrane transport.

(2) The increase of rate of transfer at the contra lateral side for the inward transport augmented the rate of change in concentration of each component. The corresponding changes in the optimal control showed a variety of changes.

(3) Present investigation must be available for evaluation of the optimality in carrier mediated membrane transport.

(Manuscript received July 10, 1996,

revised April 21, 1997) 


\section{References}

(1) Yamanobori, 1. Biological Membrane Engineering. Ed by M, Futai, Maruzen Press (1991)

(2) Y. Kagawa : Illustrative molecular biology, Nanzando Press, Tokyo (1993)

(3) V,P. Skulachev: "Membrane Bioenergetics". SpringerVerlag Press, Berlin (1988)

(4) D. J. Sanders: Theoretical. Biol, 49, 439 459 (1975)

(5) LI. Harrison \& HN. Christensen : J. Theor. Biol. 49, 439 459 (1975)

(6) M. Stuhmer: Ann, Rev, Biophys, Biophy, Chem, 20, 65 (1991)

(7) K. Miura: Biological membrane engineering. Maruzen Press, 138 (1991)

(8) S. Tamura: J, Biological, Chemistry, 264, No. 15, 8580 8584 (1989)

(9) P, C. Maloney: J, Membrane, Biol, 67, 1 12 (1982)

(10) W, L Laubinger: Biochemistry, 27, 7531 7537 (1988)

(11) H. Hirayama: IEICE Trans, Fundamentals, E76-A, No.12, 2076 2081 (1993)

(12) H. Hirayama: IEICE. Trans, Fundamentals, E77-A, No. 11, 1896 1902 (1994)

(13) L, S. Pontryagin: "The mathematical theory of the optimal process", J. Wiley Inc Press (1962)

(14) W, R. Lieb: Biophysical. Joumal, 10, 585 609 (1970)

(15) L, K. Wright: Biochim and Biophysica, Acta, 855, 391 416 (1986)

(16) J, K. Wright: Ann, Rev, Biochem, 55, 225 248 (1986)

(17) D, G Hoarse, J, Physiol, 221, pp. $311 \sim 329$ (1972)

(18) L. J. Parent: Membrane Biology. 1992; 125: 63 79

(19) D. Regen: Biochim, Biophys, Acta, 79, 151 -166 (1964)

(20) A. Kotyk: Biochim, Biophys, Acta, 135, 112 119 (1967)
Hirohumi Hirayama (Menber) was born at 1955. He received the M.D degree in Neurophysiology from Hokkaido University school of medicine in 1985. He worked as a cardiologist in Hokkaido University from 1985 to 1990 and is now an assistant professor in Department of Public Health in Asahikawa medical college. His main theme is an optimal modeling of biological system. He is a member of S.I.C.E., J.S.M.E., J. Applied Physics. Medical Electronics, J, Circulation Society.

Toshihiro Nishimura (Non-member) was born in Tokyo, Japan, in 1950 . He received the B.E. degree in electrical engineering, from the University of Oita, Oita, Japan. In 1978 he was appointed Assistant Professor of Electrical Engineering at the University of Oita. His research interests include all aspects of power electronics and power semiconductor devices, particularly those topics that combine the medical power electronics. $\mathrm{Mr}$. Nishimura is associated with the Institute of Medical Electronics, and the Faculty of Medicine, the University of Tokyo, and he is a member of the Institute of Electrical Engineers of Japan, and the Council of the Japan Society for Power Electronics.

Yuzo Fukuyama (Member) graduated Kanazawa University school of medicine at 1957. He is now a Professor of the Depart. ment of Public Health of Asahikawa medical college. He is a member of J.S. Public health. His main interest is computer analysis of patho-physiological state in Biological System. 